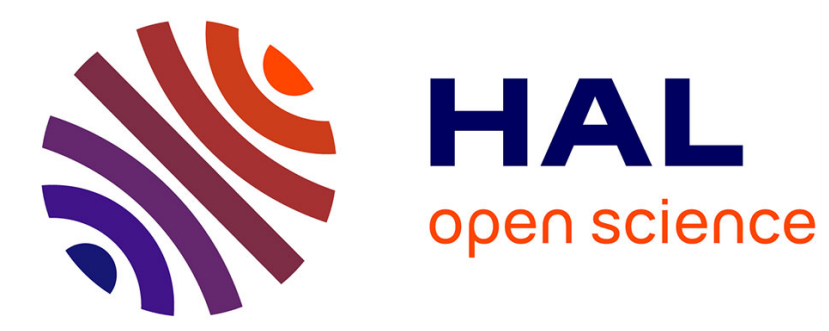

\title{
Le faste de la table dans la poésie abbasside \\ Véronique Pitchon
}

\section{To cite this version:}

Véronique Pitchon. Le faste de la table dans la poésie abbasside. Archimède: archéologie et histoire ancienne, 2021, Archimède. Archéologie et histoire ancienne, 8, pp.207-217. 10.47245/archimede.0008.var.02 . halshs-03280142

\section{HAL Id: halshs-03280142 \\ https://shs.hal.science/halshs-03280142}

Submitted on 7 Jul 2021

HAL is a multi-disciplinary open access archive for the deposit and dissemination of scientific research documents, whether they are published or not. The documents may come from teaching and research institutions in France or abroad, or from public or private research centers.
L'archive ouverte pluridisciplinaire HAL, est destinée au dépôt et à la diffusion de documents scientifiques de niveau recherche, publiés ou non, émanant des établissements d'enseignement et de recherche français ou étrangers, des laboratoires publics ou privés. 
1 DOSSIER THÉMATIQUE 1

SIUE DEUS SIUE DEA. DÉNOMINATIONS DIVINES DANS LES MONDES GREC ET SÉMITIQUE : UNE APPROCHE PAR LE GENRE

98 DOSSIER THÉMATIQUE 2

TRADITION ET TRANSMISSION DANS L'ANTIQUITÉ : RÉFLEXIONS INTERDISCIPLINAIRES

159 ACTUALITÉ DE LA RECHERCHE

QUOI DE NEUF À L'OUEST DE STRASBOURG ? KOENIGSHOFFEN :

ÉTAT DES LIEUX ET DÉCOUVERTES RÉCENTES

VARIA

195 LuCa BASILE

Forme di contatto, scambio ed interazione culturale nella tradizione vascolare campana tra VIII e VII secolo a.C.

207 Véronique PITcHon

Le faste de la table dans la poésie abbasside

\section{Cassandre HARTENSTEIN}

La période strasbourgeoise de Pierre Montet (1919-1948)

233 Anna Maria Desiderio et Arianna Esposito

Genre et mobilité à l'aune des relations socio-culturelles : I'exemple de la Campanie archaïque 


\title{
LE FASTE DE LA TABLE DANS LA POÉSIE ABBASSIDE
}

\author{
Véronique PITCHON \\ Directrice de recherches au CNRS \\ Université de Strasbourg \\ UMR 7044 Archimède \\ pitchon@unistra.fr
}

\section{RÉSUMÉ}

La poésie de la période abbasside ( $\mathrm{IX}^{\mathrm{e}}-\mathrm{XIII}^{\mathrm{e}} \mathrm{s}$ ) est I'un des fleurons de la littérature arabe. Celle dédiée à la gastronomie et à l'art de la boisson se prête particulièrement bien à une description idéalisée et imagée de l'alimentation. Elle décrit les activités les plus sensuelles de l'être humain, à savoir I'amour, la boisson, la nourriture et la table. Les poètes s'extasient sur la beauté des aliments et des plats dont on décrit la saveur, la couleur, la forme de vaisselle qui les contient. Nous avons exploré cette littérature qui exprime

\section{MOTS-CLÉS}

Poésie arabe, gastronomie médiévale, monde arabo-musulman, manuscrits culinaires. de manière métaphorique les splendeurs de la table mais qui permet aussi à I'historien de se faire une idée nouvelle de la gastronomie médiévale arabe.
The poetry of the Abbasid period ( $9^{\text {th }}-13^{\text {th }}$ centuries) is one of the jewels of Arab literature. That dedicated to gastronomy and the art of drinking lends itself particularly well to an idealized and pictorial description of food. It describes the most sensual activities of human beings, namely love, drink and food. The poets rave about the beauty of foods and dishes, with descriptions of their flavor, color, and of the vessels that contain them. This paper explores this literature which metaphorically expresses the splendours of the table but which also allows the historian to get a new idea of medieval Arabic gastronomy.
KEYWORDS

Arabic poetry, medieval gastronomy Arab-Muslim world, culinary manuscripts. 
Dans un article précédemment consacré au luxe à la table abbasside [1], nous nous étions concentrés sur le banquet de cour. Nous avions montré que la table du calife est avant tout un lieu de mise en scène et de représentation du pouvoir. Mais la table est dressée en bien d'autres endroits qu'au palais califal et elle est un lieu privilégié pour exprimer la sensualité, la beauté, le faste. La nourriture est au centre de nombreux discours qui I'analysent et la décrivent en l'investissant de valeurs multiples et en l'insérant dans des programmes narratifs multiformes. Parmi ces formes, nous avons choisi de traiter l'expression du luxe alimentaire au travers du récit poétique. On a coutume de dire que dans la littérature arabe, la poésie est bien plus qu'un genre littéraire, c'est son fleuron, un ornement de la pensée et de la conversation, une langue imagée et descriptive en même temps.

La poésie - ou le récit poétique - donne souvent une description idéalisée et imagée de l'alimentation ; elle suggère autant le manque et l'envie, que l'abondance et l'excès. La poésie arabe peint par le menu les activités les plus sensuelles de l'être humain à savoir, et en premier lieu, la boisson, l'amour, la nourriture et la table. De nombreux poètes et littérateurs arabes s'y sont essayés comme en témoigne la multitude de textes qui nous sont parvenus. Le corpus est extrêmement vaste, on parle ici non pas de quelques œuvres éparses, mais réellement de centaines de poésies écrites pendant la période antéislamique, omeyyade ou, pour ce qui nous intéresse, durant la période abbasside (IXe-XII ${ }^{\mathrm{e}}$ s.) [2]. Pour cette raison, nous nous sommes limités à la poésie du $X^{\top} \mathrm{s}$., en opérant une

[1] PitCHON 2020.

[2] BLACHÈre 1958.

[3] Les trois ouvrages ont été traduits :

- Le livre de brocart ou la société raffinée de Bagdad au Xe siècle (Al-Washshā', Kitāb al-muwashshā wa al-ẓarf wa-Izurafā'), Siham BouHLA (trad. fr.), Collection Connaissance de I'Orient ( $\left.n^{\circ} 110\right)$, Série arabe, Gallimard (2004).

- L'art du commensal. Boire dans la culture arabe classique, (Kushājim, Adab al-nadīm), présenté, traduit de l'arabe et annoté par Siham BouHLAL, Paris, Actes Sud, Sindbad, (2009).

- Annals of the Caliphs' Kitchens: Ibn Sayyar al-Warraq's sélection dans trois ouvrages traitant de la table [3], tous écrits à Bagdad : un traité du savoir-vivre et des bonnes manières d'al-Washshā' ( $\mathrm{m}$. en 936) Le livre de brocart, un traité sur l'art d'inviter et d'être invité de Kushājim (m. en 970) L'art du commensal et un ouvrage culinaire d'Ibn Sayyār al-Warrāq [4], Le livre de cuisine.

Nous traiterons de tous les aspects où s'exprime le luxe de la table visualisé au travers de la verve poétique qui permet la transposition des mots en images grâce à l'imagination et à l'expression verbale. Nous étudierons ainsi de manière systématique, les aspects matériels, humains et sensoriels liés à la table, à la consommation alimentaire et alcoolisée, en particulier le vin [5]. Mais avant cela, nous replacerons la poésie abbasside dans le contexte historique de l'émergence de la cité des arts que fut Bagdad à partir du VIIIe s.

\section{BAGDAD, CITÉ DES POÈTES}

\section{POÈTE ET MÉCÉNAT}

La capitale de l'empire abbasside va polariser les talents et devenir le berceau d'une nouvelle génération de poètes. Le calife al- Manșūr et ses successeurs, un peu pour des raisons politiques et beaucoup par passion pour l'art des vers, seront les premiers à attirer auprès d'eux les poètes. Le mécénat est assumé par le calife, mais aussi par les maires du palais et par les grands dignitaires. Le poète exerce un métier auprès d'un puissant qui le rémunère. Il peut appartenir à la classe dirigeante de la cour ou encore à une catégorie bourgeoise de commerçants ou de secrétaires

Tenth-century Baghdadi Cookbook (Ibn Sayyar al-Warraq, Kitāb al tabīkh), Nawal NASRALlaH (trad. angl.), Brill, (2007). [4] On ne sait rien de l'auteur, si ce n'est qu'il était un notable de la société bagdadienne; son patronyme, al-Warrāq, suggère qu'il vendait du papier. D'après les nombreuses mentions de personnages influents de la cour califale et des poètes cités en fin de chaque chapitre, le livre fut écrit dans la deuxième moitié du $X^{e} s$.

[5] Le thème de la boisson au travers de la poésie bachique est un thème très étudié et très prisé des historiens, qui a déjà fait l'objet de nombres d'études depuis le travail novateur mais désormais ancien de BENCHEIKH 1964. 
fortunés [6]. Cette aristocratie a une grande culture. Les poètes appartiennent à des familles intellectuelles assez diverses et fort caractéristiques du milieu bagdadien. Certains sont de hauts fonctionnaires, voire des dignitaires, comme Isḥāq al-Șābi qui se distingua à la fois comme poète et comme épistolier ; d'autres sont issus du peuple, tel Abū al-Tayyib al- Mutanabbī, le plus prestigieux panégyriste de la littérature arabe.

On se met au service d'un maître riche, exigeant et capricieux ; on est convié à l'un des cercles, salon connu sous le nom de majlis, mais on peut également être convié au repas pour l'animer en déclamant des vers, au milieu des plaisirs de la nourriture, du vin, de la danse et du chant. La poésie se prête à l'exercice du décryptage des manières de table à la cour. Le poète n'est pas un écrivain déconnecté des faits et gestes de la haute société ; il en est un maillon essentiel, un commensal recherché, parfois adulé, une véritable «star » et sa rémunération témoigne parfois de cet engouement de la haute société pour ces littérateurs dont certains ont connu une célébrité supérieure à celle des princes qu'ils servaient.

Les poètes vivant dans un état de sujétion sont présents pour encenser leur hôte mais quelques-uns cependant font un effort, sinon pour y échapper, du moins pour en limiter l'emprise. La poésie devient alors subversive, humoristique, voire caustique. Leurs voix sont reconnaissables par une liberté de ton et un accent satirique. Leurs vers pleins de dérision et de violence tranchent avec la poésie ancienne formelle et reconnue. La nouvelle poésie prend une tournure populaire, taillée pour l'amusement et la délectation d'un large public.

\section{L'ART VERBAL DE LA DÉLICATESSE}

Les comportements qui marquent le raffinement font l'objet d'une littérature particulière, l'adab, une littérature qui revêt bien des aspects mais qui, dans son sens classique, mêle l'art du bien vivre et du bien écrire, sous la forme d'une réflexion à la fois déontologique donnant une définition des valeurs et des vertus identitaires permettant de reconnaître les membres de même catégorie [7]. Mais l'adab correspond aussi à l'ensemble des vertus morales caractérisant les bonnes mœurs (akhlāq) prêtées à une nature noble et généreuse (makārim) [8]. Les qualités morales, physiques et langagières particulières qui permettent de qualifier quelqu'un de raffiné (z̧arīf pl. zurafā’) [9] sont une réalité codifiée par les sources littéraires arabes dès le IXe $s$. dont la principale à nous être parvenue est sans doute le Livre du raffinement et des raffinés [10]. Ce texte énonce les lectures, les fréquentations et les manières qui siéent à tout individu souhaitant se distinguer de l'ignorant (jāhil) et devenir un homme d'esprit ('āqil). Distinction, préciosité des manières, politesse et délicatesse de goût, recherche de la perfection dans les moindres détails, extrême raffinement dans le bien-être voluptueux sont les caractères qui distinguent le raffiné. Il veillera à ne fréquenter que des réunions d'hommes intelligents, à lire et à transmettre des poèmes, car, plus qu'un art, la poésie est une des qualités de l'homme abbasside raffiné [11].

La poésie est avant tout un art verbal; elle possède un rythme, un souffle, et propose une image subtile des codes connus qui évoque à tous les participants conviés à la table un ensemble de valeurs communes qui marque l'appartenance identitaire. Cette oralité est sans doute l'un des caractères les plus importants de la table. La voix humaine est une dimension du texte, elle participe à sa matérialité [12]. La poésie se déclame seul lorsqu'un poète veut faire connaître les nouvelles pièces de son œuvre, mais est citée également au détour de la conversation, exprimant la culture et la connaissance des codes. Cette oralité participe à l'une des qualités les plus remarquables à table, celle de l'art de la conversation. La table est en effet un lieu de plaisirs où toutes les manifestations de festivité se mêlent au plaisir intellectuel de la conversation. D'ailleurs, Kushājim citant al-Rūmī écrit dans L'art du commensal :

\section{Je suis las de toutes mes occupations}

Comme si la plus agréable était devenue la plus laide Hormis la conversation, elle seule porte un nom Qui la fait toujours neuve [13].

\section{L'ÉLOGE DU PLAISIR}

La poésie consacrée à la nourriture est une poésie non savante, dépourvue du maniérisme et de la technique de la poésie préislamique. Cette dernière s'est construite autour de thèmes classiques et s'articulait autour de genres majeurs : madīh (éloge), hijā' (satire), rithā' (élégie funèbre), fakhr (jactance ou éloge de soi), nasīb (évocation nostalgique de la femme aimée). Plusieurs de ces thèmes deviendront des
[6] Ahsan 1979.

[7] AbBÈs 2010.

[8] Balda-Tillier 2014.

[9] GHAZI 1959.

[10] Le livre de brocart.
[11] ENDERWITZ 1989.

[12] Pour la poésie médiévale, voir ZUMTHOR 1987. Sur la poésie arabe voir plus spécifiquement le travail de BonNAUD 2008, p. 81-100.

[13] L'art du commensal, p. 52. 
genres à part entière dans les siècles suivants, tels les tardiyyāt (scènes de chasse) et les khamriyyāt (poésies bachiques). Au fil des siècles, et avec I'avènement des grandes dynasties omeyyades et abbassides, si plusieurs thèmes poétiques restent majeurs comme le poème d'amour (al-ghazal), la satire (al-hijā'), l'éloge (al-madīh), la poésie bachique (khamriyya), un changement s'opère sur la manière de déclamer le poème. À I'origine, l'esthétique verbale est quelque peu secondaire mais la situation évolue rapidement. Commodité de prononciation, éloquence du vers, agrément sonore et respect de la métrique : le bon poème doit impérativement réunir ces diverses qualités.

Avec les nouveaux poètes la poésie arabe classique innove en accentuant la présence de thèmes comme le plaisir ou le mode de vie urbain, par un discours plus libre et plus en phase avec les mutations sociales de l'époque et le vécu des citadins. Parmi ces plaisirs mis en avant, il y a la nourriture, décrite dans ce nouveau genre poétique qui s'adresse à la sensibilité de l'auditeur à table. C'est une poésie de bouche et de goût qui véhicule la beauté des mets présentés sur la table et en fait la louange par le regard et la contemplation. Si l'éloge du goût se fait par le banquet, la beauté de tout ce qui l'entoure est servie par les mots déclamés par les poètes.

\section{LE RAFFINEMENT : UN LIEN IDENTITAIRE}

\section{RUPTURE AVEC LE PASSÉ ET NOUVELLES NORMES}

Un groupe social est clairement identifié au sein de la société bagdadienne ; celui des raffinés [14]. Mais attention, le raffiné n'est pas nécessairement riche ; c'est un dandy dont le milieu social est varié mais qui se distingue par sa connaissance des règles et des arts de la table : conversation, manières de manger et de boire, de partager la coupe. Al-Washshā dit : « La pauvreté n'exclut pas du raffinement les hommes d'éducation » et citant un poète dont il ne donne pas le nom, il poursuit :

« L'homme peut atteindre la noblesse, son manteau usé, l'échancrure de sa chemise rapiécée [15] » .

Les comportements marquant le raffinement à table sont hautement codifiés. Les raffinés doivent privilégier certains aliments et s'abstenir d'autres afin de développer un modèle gustatif qui soit une expression de leur délicatesse et de leur art de vivre.

L'invitation au banquet est formelle ; il reste un lieu ouvert où gravitent un ensemble de personnes qui s'invitent sans avoir été conviées, mais qui, d'une certaine façon, mettent en lumière la générosité de I'hôte. On a, par exemple, toute une littérature sur un groupe particulier de la société qui s'organise en corporation, celui des pique-assiettes [16]. Abū al-Faraj nous donne une idée de la sociologie des participants au banquet grâce à ce poème [17] :

\section{Je vins plus tôt que le chant des oiseaux,}

Le soleil comme la dague était encore caché,

Je vins avec un groupe de commensaux prometteurs, Nobles, gloutons, opportunistes, héritiers du trône, intelligents et bons à rien

Nous arrivâmes comme une nuée de sauterelles, vêtus avec élégance, le plus admirable des groupes,

Guidés par l'odeur qu'exhalaient les fèves,

Dieu nous bénisse, plus besoin de chercher à manger. Une gazelle exquise nous accueillit

Avec un vin rouge aux reflets roses comme ses joues, offert généreusement, et remplissant nos verres.

Ces pratiques tranchent avec celles qui forgent l'idéal imaginaire de la société arabe, frugalité, rigueur et pauvreté digne de la société bédouine, mère et inspiratrice de l'unité arabe telle qu'elle se conçoit. Pour le bédouin, la nourriture est simple, fondée sur un nombre restreint d'ingrédients produits dans un milieu aride et difficile. La cuisine bédouine est avant tout un mode de subsistance qui laisse transparaître une vie de privations et qui, curieusement, constitue un fantasme des origines pour sédentaires.

Ce n'est pas un hasard si les aliments bannis par les raffinés sont ceux privilégiés par la société bédouine. Al-Washshā' les énonce dans Le livre de brocart [18] :

«Les raffinés ne mangent ni friture, ni poisson haché, ni pâté de poisson, ni petits poissons séchés, ni kāmikh [19], ni salaisons. Manger de ces choses-là est une énormité à leurs yeux [...]. Les raffinés ne mangent ni sauterelles, ni langoustes car elles ressemblent au sexe des animaux [...]. Quant aux plantes aromatiques, ils les servent à table, en donnent à manger aux esclaves, mais n'en mangent pas beaucoup eux-mêmes 
et jamais un plat entier. Ils goûtent à une petite quantité de menthe, mais évitent les feuilles de chicorée et la cuscute, en raison de leur tempérament froid, radis et cresson pour leur puanteur, poireaux et oignons pour leur odeur [20], luzerne et mélilot pour leur rudesse et aussi parce qu'ils colorent les dents et les gencives en vert, altèrent et corrompent I'haleine. Qu'un seul morceau d'ail tombe dans la marmite, ils ne goûteront jamais au plat ; de même pour l'oignon, ils n'y toucheront jamais. Ils ne prononceront jamais le mot persil à cause de la première partie du nom [21] et la laideur de l'expression [...]. Ils répugnent à toucher les carottes, ne les regardent ni n'en mangent ».

Les manières de table constituent un code de bonne conduite qui distingue le zarîf de la plèbe ; elles sont si importantes que les moindres détails y sont invoqués et consacrés [22]. L'hygiène par exemple est une condition obligatoire de la participation à table. L'hôte est parfumé, bien habillé, il respecte le cérémonial du lavage des mains avant et après le repas. Le repas se conclut par un ensemble de rituels ; I'un consiste en I'usage du cure-dent [23], dont la fabrique et l'usage sont décrits dans les traités d'hygiène et les livres de cuisine et dont l'importance n'échappe pas aux poètes, dont Kushājim [24] :

Le cure-dent que je vis, il le tenait au dîner entre ses doigts,

Comme une flèche qu'une main experte aurait aiguisée.

Dans sa main, le pic était comme une svelte gazelle dans la steppe.

La main et la steppe dans un mouvement réunies, Le pic et la gazelle, qui se balançaient de manière égale.

[20] Selon un hadîth cité par Ibn Khaldūn : « le Prophète ne mangeait ni ail, ni poireaux, ni oignons, parce que les anges venaient le visiter et qu'il s'entretenait avec Gabriel et il ne voulait pas qu'ils fussent incommodés » (Prolégomènes, Slane, 1863, tome I, p. 187).

[21] Tarreh en persan, qui est en fait une variété de poireau, dite Nabatéenne. Tarr peut être le début de la racine du verbe « fendre » ou de « chasser quelqu'un ».

[22] L'art du commensal, p. 193-194.

[23] Appelé khilāf, miswāk ou siwāk, son usage commun vient de la tradition prophétique qui dit «Purifiez votre bouche, car par elle passe la purification divine. » C'est le bois dont ils sont fabriqués qui les distingue, (khilāf en saule, miswāk et siwāk, bois d'araq, Dozy, Supplément, t. 1 p. 109, 388, 747). [24] Kitāb al tabīkh, p 493.

[25] En voici I'une des compositions données dans le Kitāb al-Tabīkh de Sayyār al-Warrāq (trad. N. Nasrallah), p. 495 : « Prends des clous de girofle, de la cannelle, de la cardamome
Il en va de même du lavage des mains, pour lequel on utilise le ūshnān [25], composé alcalin délicatement parfumé se substituant au savon grossier et qui a aussi droit à une versification d'Ibrāhīm al-Mahdī, prince abbasside, roi des gourmets [26] :

Le pot en cristal qui contient le ushnān est gravé dedans et dehors On n'y voit les bêtes et les oiseaux de lui émanant un subtil parfum Qui sent comme le musc éclaboussant le cou, et semblable à des perles de camphre Comme de la soie douce et luxuriante au toucher,

Le mahlab placé au centre

Débordant d'un liquide d'huile de moringa [27] et de fleurs odorantes, aux senteurs du vin Plus aromatique que l'ambre posée sur la poitrine, à l'odeur plus forte que le parfum des princes.

\section{LES PARTICIPANTS}

La pratique du manger ensemble est une norme sociale; on ne mange pas seul mais en groupe pour une célébration commune de la beauté autour des plaisirs esthétiques. La célébration de la beauté est ritualisée par la présence d'invités connaissant les codes du comportement et du savoir-vivre.

À tout seigneur, tout honneur ; commençons par l'hôte dont Kushājim nous rappelle les devoirs dans une longue tirade s'étalant sur plusieurs pages de son ouvrage [28]. Il doit être équitable en matière de compagnie, refuser distance et retenue, être à son aise et parfaitement détendu et témoigner d'une généreuse amitié et d'une compagnie parfaite. Il devra éviter de mettre son invité mal à l'aise. L'hôte ne doit pas verser à boire à son invité de ce dont il ne boit pas. Il doit faire venir une boisson que l'invité trouve bonne et convenable pour son corps, il doit lui servir le vin dont il dispose chez lui. Il doit être généreux et emmener la cruche qui porte encore son sceau de terre, prouvant qu'elle est nouvellement ouverte.

et du cubèbe, une part de chaque. Prendre aussi à part égale du mastic, des feuilles de cyprès de la résine de storax. Tu as aussi besoin de trois parts de marjolaine, de cinq parts d'argile blanche de La Mecque et de I'alcali de Bāriqa (un endroit près de Kūfa en Irak), le double ou le triple de la quantité d'argile. Prépare aussi du riz qui a été trempé, séché, écrasé et tamisé, la même quantité que l'alcali. Réduit en poudre chaque ingrédient séparément puis mélange-les, si Dieu veut ».

[26] Kitāb al tabīkh, p. 497.

[27] L'huile de ben ou huile de moringa a été utilisée pour des onguents et des préparations, depuis l'époque des Égyptiens. L'huile d'une couleur jaune doré a un goût plaisant et sa qualité est comparable à celle de I'huile d'olive. L'huile de ben a été utilisée depuis des millénaires en tant que base pour les parfums et elle continue à jouer ce rôle encore aujourd'hui.

[28] L'art du commensal, p. 65-73. 
La tenue vestimentaire fait elle aussi I'objet d'une codification. Les tuniques doivent être légères, les chemises épaisses en lin de qualité supérieure et de couleur naturelle, les robes à manches provenant du Khorasan ou d'Alexandrie, et de tous les lieux où I'on fabrique des étoffes de qualité supérieure. Les voiles sont en lin fin et soyeux, les manteaux fourrés, les capuchons en étoffe de soie écrue et de couleur unie ou en lin brodé. Les manteaux de trois quarts en étoffe unis et brodés, venant de Nishapur ou du Yémen, sont en soie, brodés et ornés de dessins et de figures de Sus, les capuchons bleus de Khumis etc. En revanche, il n'est pas de bon ton de porter des habits de couleur criarde, plongés dans des bains de parfum sentant le safran, comme la soie mélangée jaune et le lin broché parfumé d'ambre, car ce sont là des vêtements propres aux femmes, aux esclaves-chanteuses et aux servantes. De même, les raffinés adoptent les sandales d'Abyssinie, des modèles en peau épaisse doublée de peau à poil du Yémen, les souliers en cuir fin, les bottines serties de pierreries, en particulier, celles où le rouge est superposé au noir et le jaune au noir. Ils portent un type de bottines particulières à bords retroussés vers l'arrière et à lacets. En matière de ceinture, ils portent des cordons en soie écrue, à bordure de coton et des cotons brodés d'Arménie.

D'autres participants sont eux aussi acteurs du spectacle qui se met en place. Danseuses, chanteurs et la figure ambigüe de l'échanson sont là pour rappeler que le poète n'est pas tout à fait détaché du ghazal, genre poétique chantant l'amour. Il existe toute une littérature sur l'échanson chargé de servir le vin. C'est souvent un jeune homme qui cristallise de magnifiques vers poétiques à la dimension érotique, ne laissant aucune place aux doutes quant à des pratiques homosexuelles. Les cuisiniers, les serveurs, les esclaves également loués par les poètes participent au bon fonctionnement du repas.

\section{LES PARADOXES}

Ibn al-Washshā' insiste sur le fait que la frugalité convient au raffiné bien plus que la gloutonnerie [29]. Mais cette frugalité, bien que recommandée, ne cède en rien le pas à la consommation de mets coûteux ou rares, élégamment présentés et souvent en proportions bien peu propices à la frugalité, toute tentative d'avarice et de restriction étant systématiquement sanctionnée par les partisans de l'art du raffinement. La table est donc le lieu paradoxal où la norme du bon goût, par essence marquée par la restriction, est transgressée par la quasi-obligation d'abondance. Les règles imposées aux Raffinés : frugalité, contrôle de soi, raffinement sont oubliés au profit de l'abondance et de l'abus de nourritures, du vice (échanson, présence féminine) et au gré de l'avancement du repas et de la consommation d'alcool, effacées par un étiolement des manières et du « self-control ».

Pour les Raffinés, toute tentative de lésiner est une transgression du bon goût et de l'élévation de l'esprit. Seul compte ce qui est beau, ce qui est bon et ce qui est cher. L'idéal alimentaire des poètes modernes de Bagdad est bien éloigné de l'austérité prônée par les poètes de la période antéislamique qui chantaient la frugalité et la rigueur du régime des bédouins basé sur la consommation de dattes et de lait caillé.

\section{NOURRITURES, ESTHÉTIQUE ET POÉTIQUE DE LA TABLE}

La table inspire le poète et selon Ibn Rashīq la bonne chère et le bon vin, consommés en écoutant des chants harmonieux, sont propices à la poésie [30]. Le répertoire de la poésie arabe comporte de petites merveilles où les poètes réussissent la prouesse de donner à voir toutes les qualités de la nourriture. Leur ingéniosité réside dans leur capacité à sublimer en mots les mets les plus simples. Leur créativité rend les aliments les plus ordinaires appétissants, désirables, recherchés, luxueux. Cette transformation s'opère par le biais de la métaphore alimentaire, une isotopie alimentaire qui dicte à l'auteur des métaphores gustatives « douce comme le miel » et visuelles «blanche comme la lune », etc. On en trouve certains exemples dans ce poème d'Abū al-Faraj sur les fèves [31] :

Belle fève pelée, musc de la terre, comme le miel, tendre et juteuse

Ton lustre embellit le plus sensible des regards, il guérit des peines et des griefs du cœur

Ta place, si fraîche et loin de la chaleur qui fait fondre les pierres, rafraichissante comme un souffle léger en été

Tel un collier de pierres précieuses pas encore formé au cou d'une vierge délicieuse,

Des perles enfermées dans une coquille d'émeraude Cueillies aujourd'hui, par une seule main, sans un jour de retard,

Plus douce que le sommeil après une nuit blanche et que la promesse d'un rendez-vous galant.

[29] Le livre de brocart, p. 178-180.

[30] Ibn Rashīq, Al-'Umda fi șinā'at al-shi'r wa-naqdih, t. II, 211.

[31] Kitāb al tabīkh, p. 230. 
Les figures de style sont métaphoriques, elles consistent à localiser dans la bouche, sous la langue, le plaisir de la consommation. Ce type de métaphore s'applique à la vue, à l'odorat, au goût, mais les autres sens comme le toucher et l'ouïe font aussi partie du banquet. 'Uthmān ibn Șadaqa ibn Waththāb évoque ici cette sensualité [32] :

Le vin semble rosé dans le verre, ce cher enfant au père disparu depuis si longtemps [33]

Le miel, sur les mains qui le pétrissent et le mélangent, est onctueux et épais

Belle journée à toi mon ami, sois mon invité, et ce que je vois, vois-le aussi.

\section{INGRÉDIENTS}

Tous les ingrédients sont évoqués, en tant que tels, ou combinés dans un plat. Les comparaisons communes évoquent les matériaux précieux, les couleurs et les parfums. Nous allons, dans un premier temps, passer en revue certains aliments et plats les plus significatifs. Ce sont les plus connus, les plus appréciés, donc les plus sujets à l'emphase des poètes.

Le monde végétal est à l'honneur. Chaque légume ou fruit a droit à son couplet. L'aubergine, par exemple, sous la plume de Kushājim, prend une dimension paradisiaque [34] :

L'ignorant me réprimande quand je lui révèle l'aubergine Alors que le docteur lui-même ne cessera jamais d'en manger

Je lui dis, elle est comme l'amant qui a trouvé l'amour Elle est comme le rival qui se tient face à moi

Si jamais tu en manges dans mon dos, ton allié sera fâché.

L'aubergine a le goût de la salive qu'un amant généreux offre sans réserve

Une perle cachée dans une robe noire surmontée d'une tige vert émeraude

Au goût sans nul autre pareil, qu'elle soit cuite vite ou lentement mijotée.

Désirant ardemment cette petite merveille, les plus spirituels des invités s'empressent de la consommer

Seuls les fous ne l'aiment pas, mais les plus intelligents l'adorent.

[32] Kitāb al tabikh, p. 225.

[33] Comme la grappe, le père, l'est pour le vin, le fils.

[34] Kitāb al tabīkh, p. 228.

[35] Kitāb al tabīkh, p. 281, (poème d'Al-Hāfiż Muhammad bin al-Wazīir).

[36] Abu al-Hasan 'Alī ibn al-Husayn ibn 'Alī al-Mas'ūdī (896-956 ap. J.-C. environ, 283-345 après l'Hégire) fut un historien et
Le cas de l'aubergine est instructif ; bien qu'on la trouve communément dans les manuels culinaires, I'opinion médicale générale décrit ses effets nuisibles sur les corps causés par la production de bile noire. Bien que jugée néfaste, elle est largement utilisée. Le paradoxe est donc que ce légume, tant apprécié des gourmets, est universellement décrié par les médecins qui s'empressent cependant de le consommer quand ils sont à la table des princes.

Le poète n'oublie pas de chanter les vertus médicales des plats, montrant l'importance des considérations diététiques et louant ainsi la présence des médecins de la bonne société à la table, comme pour ce plat de rỉbāsiyya, un ragoût de viande et rhubarbe, dont le goût correspond en tous points à l'appétence marquée des Arabes pour les plats aux saveurs acides et sucrées [35] :

Lorsqu'en été tu meurs d'envie de manger un plat, Un de ceux qui supprime la bile noire et la bile jaune, Prends un poulet et coupe-le en morceaux

Lave-le à l'eau et lave-le encore

Mets-le dans le pot et fais-le chanter à I'huile fraîche et claire comme l'eau.

[...]

Et quand le plat est prêt et qu'il est succulent, pur et brillant,

Les amandes ajoutées, laisse-le sur les charbons ardents. La cuillère dans le bol, propre et brillante comme une perle,

Arrosé d'eau de rose dont l'odeur se répand lorsque tu le consommes,

Et quand c'est fait, le meilleur des hommes, redouble son plaisir avec un verre de vin vierge.

L'asperge quant à elle se pare de toutes les vertus, qu'elles soient diététiques ou symboliques, Mas'ūdī [36], citant Kushājim, nous dit :

Nous possédons des lances dont la pointe se recourbe, Elles sont tordues et tressées comme une corde, mais belles et sans nœud.

Leur tête est proéminente sur leur tige, Plantées droites dans le sol comme des piliers, Elles ont été habillées par la main du Créateur unique et éternel,

géographe arabe, connu comme «l'Hérodote des Arabes ». Il fut l'un des premiers érudits à combiner l'histoire et la géographie scientifique dans un immense ouvrage, le Murüj adh-dhahab wa ma'ādin al-jawhar (Les prairies d'or et les mines de gemmes). Nous citons ici la version bilingue arabe-français, publiée entre 1861 et 1877 (Publication de l'Imprimerie Nationale, Charles Barbier de Meynard et Abel Pavet de Courteille), p. 399. 
D'une étoffe soyeuse, semblable à celle qui recouvre le manteau.

\section{[...]}

On croirait voir une étoffe de soie. Ah! Que n'en ontelles aussi la durée!

Elles pourraient être le chaton de bagues de perles, Un jus appétissant se répand sur elles.

Il fait tout autour d'elles le flux et le reflux, I'huile les recouvre d'un tissu d'écume,

Et en s'infiltrant dans leur tige, elle forme des tresses d'or et d'argent.

Un dévot, un grave docteur, en voyant ce plat délicieux, se prosterneraient avec convoitise et rompraient le jeûne.

L'ensemble des expressions de la métaphore sensorielle est donné dans ce poème parlant d'un simple plat de carottes servies froides avec des herbes aromatiques et des épices, déposé dans un plat choisi à dessein : même la vaisselle n'échappe pas à la verve poétique. C'est l'ensemble de ce qui constitue la table qui est loué et chanté par Kushājim [37] :

Dinars de cornaline et d'or dans une vaisselle si délicate, qu'elle pourrait fondre et couler

Irradiant d'un lustre de cornaline et brillante comme la perle

Dans un récipient harmonieusement choisi, ici rassemblées et là-bas dispersées

Les épices exhalent un doux parfum tel le fumet d'un vin délicieux

Le dessus est semblable à des perles et de l'argent intercalés de pierres précieuses

Que le cuisinier a délicatement façonnées, Un plat délicieux tant en parfum qu'en goût

La rue saupoudrée telle la turquoise, d'un vert éclatant Se mêlant au murrī [38] et à l'huile d'olives,

Elle monte et descend dans un éclat luisant.

Les fruits, comme les dattes, l'orange et la banane, telles les plus belles offrandes de la nature ont aussi droit à des déclamations enflammées, comme celle d'Ibn Rūmī [39] :

La banane, par Dieu,

Ce mets délicieux

Courtise nos entrailles.

Sa saveur sans pareille,

Son haleine embaumée

Hausse l'âme pâmée

Au-delà du plaisir.
On croit rêver sans mentir

Que l'insinuation du fruit dans mon méandre Vaut la défloraison d'une vierge très tendre.

\section{PLATS ENTIERS ET VIANDES}

Les viandes les plus nobles, chevreau et antilope (ou gazelle) sont des mets très recherchés et parmi les plus chers que l'on puisse offrir à ses invités. Elles sont servies en sauce dans des ragoûts épicés qui portent le nom du végétal qu'ils contiennent. Mais pour Kushājim, les viandes rôties et grillées ou embrochées sont à l'honneur [40] :

Pour mes honorables invités, j'ai du pain, pur, craquant et bien levé.

Déposé sur le plat, on croirait voir la lune resplendissante de beauté.

Et pour l'accompagner, des côtelettes d'agneau, en abondance,

Épicées, vinaigrées, odorantes et safranées.

Un excellent cuisinier les a préparées, marinées aux épices, au sel et au murrī

Mélangées aux aromates durant toute la nuit, Conservées ainsi, au point du jour il les préparera.

Il allumera le four, elles deviendront brillantes sous l'effet de la flamme

Il scellera le plat et les laissera cuire

Jusqu'à ce qu'il soit sûr que la viande tombe de l'os.

Une pluie de coriandre il ajoutera, éthéré et brillant avec du safran.

Elles seront semblables aux dattes, fraîches, craquantes et mûres

La poésie de table, par la vertu des métaphores alimentaires, conduit à une assimilation du poème en un plat qui se déguste. Les métaphores esthétiques sont nombreuses et mettent à contribution l'odorat et la vue. Parfum, couleur, texture sont évoqués comme l'argent, I'ambre, l'or, les perles, la soie, les pierres précieuses. La métaphore de la pierre précieuse, et en particulier de l'émeraude et des perles de nacre, est très usitée, par exemple dans cette description poétique de d'Ibrāhīm al-Mahdī d'une zīrbāja, zīrbāja, plat de viande au vinaigre [41] :

Le cuisinier s'approche tenant un plat rond comme un soleil levant

Rempli de zīrbāja , parfaite et dressée avec dextérité, Comme un collier qu'un orfèvre aurait paré de pierres précieuses

[39] Le diwan de Bagdad, p. 76.

[40] Kitāb al tabīkh, p. 365.

[41] Kitāb al tabīkh, p. 273. 
Des perles d'oignons alternant avec de la courge émeraude pure et immaculée,

De délicates carottes semblables à des feuilles d'or le plus pur, la ceinturent

Colorées avec du safran, on les prendrait pour des anémones

L'aubergine marque sa présence, safranée du rouge éclatant de la cornaline

Dispersant des arômes de camphre, de musc et d'ambre, succulente comme du miel pur.

La blancheur de la lune est une métaphore courante, employée pour de nombreux plats. Cette couleur associée à la pureté, à la brillance très recherchée, est mise en valeur pour de nombreux plats et sauces. Le plat le plus chanté de tous est sans doute la madira [42] :

La madira dans un repas, c'est la lune dans la 14e nuit du mois. Elle brille sur la table comme une lumière qui dissipe les ténèbres,

Comme le disque argenté de la lune qui apparaît à travers les nuages.

Elle remplit une jatte faite d'onyx du Tihamah [43]

Les pâtisseries représentent le summum de l'art de la cuisine abbasside. La saveur sucrée symbolise la fête et donne lieu à la plus grande créativité culinaire. Les douceurs sont destinées à la dégustation mais aussi à la vue et se présentent sous des formes variées, gâteaux, nougats, pâtes de fruits et sirops colorés aux multiples ingrédients. Les plus connus sont le fälūdhaj, fait de poudre d'amandes, de sucre, d'eau de rose, d'épices. Les nougats tel le lawzinaj, sont faits d'amandes, de mie de pain, de sirop de rose, de sucre et d'huile de sésame, ou encore le nățif, fait d'un mélange de miel purifié, additionné de sucre blanc dont l'éclat est exalté par des blancs d'œufs battus en neige, mélangé aux amandes, aux noix, aux graines de sésame ou aux pistaches. Le zalābìya, une pâte remplie d'amandes pilées et de sucre, parfumée à l'eau de rose, au musc ou au camphre, avec du safran, trempé dans du miel qui lui apporte une brillance et en exalte la beauté et la délicatesse comme le chante ce poète anonyme cité dans un livre de cuisine [44] :

Pour le dessert je prends un zalābìya rond dans son treillis

Blanc et jaune et coloré, frit dans la meilleure huile de sésame

Doux au toucher, coulant de miel mādhī [45]

Comme de la cornaline disposée en rangées ou des tubes d'or pur emmaillés

Enlacés l'un dans l'autre, comme brodés de la plus belle des soies

Caché sous le sucre, comme un cloître pour des yeux indiscrets

Doux et délicat sous la dent.

L'arrangement des pâtisseries sur le plat est graphique et symétrique. Les gâteaux sont empilés de manière à former des pyramides. Arrivant en fin de banquet, dans une ambiance où l'alcool a fait effet, leur beauté et leur goût hors du commun, sont sublimés par les vers d'Ibn Rūmi [46] :

Puis voici les crèmes d'amande farcies au sucre ; Quel ciel bienfaisant répand cette pluie, quelle terre fortunée en est arrosée!

Qu'elles sont belles sur la table, dans le beurre et la saumure qui grésillent sur les bords.

Nous épluchons la peau qui recouvre la chair des amandes,

C'est de l'argent que nous dégageons d'une feuille d'or Et les hachis tout dorés de jaune d'œuf qui leur font comme un vêtement, une parure.

Puis viennent les kataifs délicieuses qui charment le palais et flattent le gosier,

Le sourire épanouit les visages à la vue de ce beau sucre candi qui le recouvre

Et forme avec le beurre une rosée de larmes

\section{BOISSONS: LE POÈME BACHIQUE}

En théorie, I'Islam interdit la consommation d'alcool [47] mais à l'époque abbasside cette recommandation est peu respectée. Sa consommation est si abondante et si commune qu'elle semble relever plus du simple plaisir que de la transgression. Si le Coran en interdit clairement la consommation, il convient en même temps de noter qu'à plusieurs reprises il promet au croyant pieux et pratiquant qu'il pourra

[42] Kitāb al tabïkh, p. 302.

[43] Région au sud-ouest de la péninsule Arabique, bordant la mer, dont on extrayait un très bel onyx.

[44] Kitāb al tabīkh, p. 417.

[45] Le meilleur des miels, blanc et délicat.

[46] MAS'ŪDI, t. 8, p. 239.

[47] Le vin est interdit ; Sourate 5, v. 90 et 91 : « Ô croyants ! Le vin, le jeu de hasard, les pierres dressées, les flèches de divination ne sont qu'une abomination, œuvres du diable. Écartez-vous en, afin que vous réussissiez » et « Le diable ne veut que jeter parmi vous, à travers le vin et le jeu de hasard, l'inimitié et la haine, et vous détourner d'invoquer Allah et de la prière. Allez-vous donc y mettre fin ? ». Mais dans la sourate XVI, verset 69 , le vin est plutôt célébré comme un signe de la faveur divine envers I'humanité. La littérature sur la consommation d'alcool est très abondante; on pourra consulter par exemple : BARBOUCHI, Sarra, « Vin et ivresse dans Qutb al-Surūr fĩ Awsāf al-Anbidha wa-I-Khumūr ( $\mathrm{X}^{\mathrm{e}}-\mathrm{XI} \mathrm{I}^{\mathrm{e}} \mathrm{s}$.) », Synergies Monde arabe 6, 2009, p. 249-262. 
le consommer au paradis [48]. Les poètes se sont emparés du thème du vin et de l'ivresse à la fois dans une description transgressive et humoristique, mais aussi en créant les plus beaux morceaux littéraires véhiculant l'image de la beauté et du plaisir. La douceur du vin et l'ivresse qui en découle est un avant-goût de la volupté sensuelle qui suivra le moment des retrouvailles au paradis.

Les boissons alcoolisées et en particulier le vin et l'ivresse sont des thèmes très prisés des poètes profanes [49]. Ici aussi on retrouve une rhétorique de la description des qualités visuelles, telles la couleur, I'assimilation des reflets aux métaux précieux mais également sensorielles. Rien n'excède en quantité le nombre de poèmes sur le vin. La poésie bachique est de loin la plus prolifique du monde médiéval musulman. On y retrouve tous les thèmes, comme la transgression religieuse, traités par le biais des métaphores humaines. Dans les poèmes à boire le vin occupe une forte charge symbolique, il s'humanise, acquiert la vie, donne de la fraternité [50].

Le thème de la bière dans la poésie reste inexploité. On boit de la bière très légèrement alcoolisée et pétillante, préparée à base d'orge fermentée parfumée aux épices et au miel, rafraichie avec de la glace, appelée fuqqā` [51]. La couleur, le parfum, le goût subtilement épicé sont exprimés avec verve par le poème d'Ibn Mu'ttaz cité par Kushājim [52] :

Faite du cœur du blé le plus pur, brillante comme l'argent,

Aussi odorante que le meilleur bois d'aloès de Mandal [53]

Elle délivre son parfum et ton esprit en meurt d'envie Aussi délicieuse que la salive du baiser des amants, Versée dans la bouteille, même le plus compétent croirait que c'est du bois de santal.

Aux odeurs de musc, de sukk [54] et d'ambre mélangés à l'essence de girofle

[48] Coran, 56, v. 17-18.

[49] HURE 1983.

[50] Je ne m'attarderai pas sur ce thème car il a été traité en détail dans la littérature notamment dans l'article fondateur de J. Bencheikh, puis dans un travail renouvelé par Mary Bonnaud. Tous deux analysent le thème de la poésie bachique au travers de I'œuvre d'Abū Nuwas. Je mentionnerai également les travaux de Bruno Paoli qui explore plus largement le thème de la poésie bachique : PAOLI 2009b.

[51] Il existe un débat sur cette boisson, qui selon certains (N. NASRALLAH, Ibn Sayyar al-Warraq. Kitab al tabīkh, p. 454) est une boisson non alcoolisée tandis que pour d'autres elle
Un tel breuvage étanche la soif avant le repas

Et tel l'électuaire, il éloigne la bile jaune

Pris avec de la glace, il guérit de la gueule de bois

Première action du matin avec un rapide repas

Comme l'eau du paradis, il décante dans le verre, surmonté d'un filet tressé de perles,

Fraîche comme des fils brillants finement entremêlés.

\section{CONCLUSION}

Durant la période abbasside, époque fastueuse de l'empire, en particulier à Bagdad, certaines catégories de population vivent dans un raffinement extrême qui va donner aux poèmes d'un nouveau genre une place de choix dans la littérature. On chante les plaisirs en général, le libertinage, le vin et l'enivrement, la beauté sous toutes ses formes et bien sûr la nourriture occupe une place prépondérante. Tous les us et coutumes alimentaires, les plats délicieux, les mets somptueux sont loués et versifiés à l'infini.

Nous avons mis en évidence qu'il existe une rhétorique du beau et de la beauté fondée sur les plaisirs sensoriels, le goût et l'odorat, sens primaires invoqués pour l'art de manger, la vue pour la couleur et le décorum, le toucher pour les textures. Bien sûr, à la table l'ouïe est sollicitée par la conversation, le chant et la poésie qui sont déclamés tout au long du repas. On peut comparer ce banquet sensoriel au banquet du paradis. Si le Coran met en garde contre les dangers du vin, il convient en même temps de retenir qu'il en fait un des délices, placé, au même titre que les houris (vierges du paradis) et autres douceurs célestes, en particulier alimentaires, au cœur de la promesse paradisiaque.

Ce paradis donne une image d'un extrême raffinement, habits de soie et de brocarts, vins doux et sucrés, nourriture succulente, variée et abondante, une image que cette société huppée tente de reproduire sur terre, bien peu pressée de vérifier les préceptes divins.

contient de l'alcool. Le fait que les poètes la vantent pour soigner la gueule de bois, semble accréditer la thèse d'une boisson non alcoolisée. Cependant, si elle est pétillante, cela indique que le processus de fermentation est enclenché et donc qu'elle doit être au moins légèrement alcoolisée. [52] Kitāb al tabīkh, Ibn Mu'ttaz cité par Kushājim, p. 458. [53] Ville indienne du Rajasthan célèbre pour son bois d'aloès.

[54] C'est une pâte noire dont l'ingrédient de base est le rāmak qui est un mélange de noix de galle et de dattes fraîches. Le rāmak est long et compliqué à faire. On en fait de petits disques qu'on enfile sur un fil et qu'on laisse sécher à l'ombre et qu'on utilise pour préparer le sukk. 


\section{Sources}

Al-Mas'ūdi, 1861 - 1877, Muruj adh-dhahab wa ma'adin al-jawhar (Les prairies d'or et les mines de gemmes), Version bilingue arabe-français, (Publication de l'Imprimerie Nationale, Charles Barbier de Meynard et Abel Pavet de Courteille). Al-Washshā', 2004, Kitāb al-muwashshā aw al-ẓarf wa-l-ẓurafā', Le Caire, (1953). Trad. fr. : Le livre de brocart, trad. et notes, Siham Bouhal, Paris.

Al-Qayrawānī, Ibn Rashīq, 1981, al-'Umda fị Maḥāsin al-Shi'r wa 'ādābih wa Naqdih. Ed. Muḥam- mad 'Ab al-ḥamīd. 5th ed. Beirut: Dār al-Jīl.

Ibn Sayyar al-Warraq, 2007, Kitab al tabīkh, "Annals of the Caliphs' Kitchens", Nawal Nasrallah (trad. angl.), Leiden. Kushājim, 2009, Kitāb al- nadīm, Bulaq, Le Caire (1928), L'art du commensal, trad. et annoté par Siham Bouhal, Paris.

\section{Articles}

Аввѐs, Makram, 2010, «L'adab et la formation de l'homme », La civilisation arabo-musulmane au miroir de l'universel : perspectives philosophiques, Paris, UNESCO, p. 29-40.

Ahsan, Mohammed, 1979, Social life under the Abbasids, 170-289 AH, 786-902 AD, London - New York, p. 157-163. Balda-Tillier, Monica, 2014, « Zuurafā' : les Raffinés de l'islam classique », dans Houari Touati (dir.), Encyclopédie de I'humanisme méditerranéen [http://www.encyclopedie-humanisme.com/?Rhetorique-dans-la-tradition].

Bencheikh, Jamel, 1964, « Poésies Bachiques d'Abū Nūwās », Bulletin d'Études Orientales 18, p. 784.

BlACHère, Régis, 1958, « Un jardin secret : la poésie arabe », Studia Islamica 9, p. 5-12.

Bonnaud, Marie, 2008, La poésie bachique d'Abū Nuwās - Signifiance et symbolique initiatique, Pessac.

Enderwitz, Suzanne, 1989, « Du Fatā au Ẓarīf, ou comment on se distingue ? », Arabica 36 (2), p. 125-142.

FAHD, Toufik 1977, Matériaux pour l'histoire de l'agriculture en Irak : al-Filāha n-Nabațiyya, Wirtschaftsgeschichte des Vorderen Orients in islamischer Zeit. I. Handbuch der Orientalistik, Erste Abteilung, VI. 6, Teil I, Leiden - Köln.

GhazI, M’hamed Ferid, 1959, « Un groupe social : "Les Raffinés" (zurafā') », Studia Islamica 11, p. 39-71.

GhersetrI, Antonella, 2004, «En quête de nourriture - Étude des thèmes liés aux pique-assiettes (țufayliyyūn) dans la littérature d'Adab », Al-qantara 25, p. 433-452.

HuRÉ, Jacques, 1983, «Le thème bachique en Islam au Ve siècle de l'hégire, à Ishbilya (Andalus) et Nishabur (Khorasan) », dans Max Milner \& Martine Chatelain-Courtois (éd.), L'imaginaire du vin, Marseille, p. 87-95.

Paolr, Bruno, 2009a, «Deux études sur la poésie bachique arabe : 1. Les précurseurs d'al-Hīra », accès 10/08/2020, [https://halshs.archives-ouvertes.fr/halshs-00366836].

Paoli, Bruno, 2009b, «Marchands, taverniers et échansons : étrangers et gens du Livre dans la poésie bachique arabe », accès 10/08/2020, [https://halshs.archives-ouvertes.fr/halshs-00366830].

Prтchon, Véronique, 2020, «Luxe (taraf) et raffinement (zarf) à la table abbasside », Archimède, Archimède. Archéologie et histoire ancienne 7, p. 327-338.

DOI : 10.47245/archimede.007.var.04

Vuong, Hoa Hoï \& Megarbane Patrick, 2008, Le diwan de Bagdad. Le siècle d'or de la poésie arabe, Paris. Zumthor, Paul, 1987, La lettre et la voix, Paris. 